\title{
Gewinner des Wissenschaftspreises Klinische Pädiatrie stehen fest
}

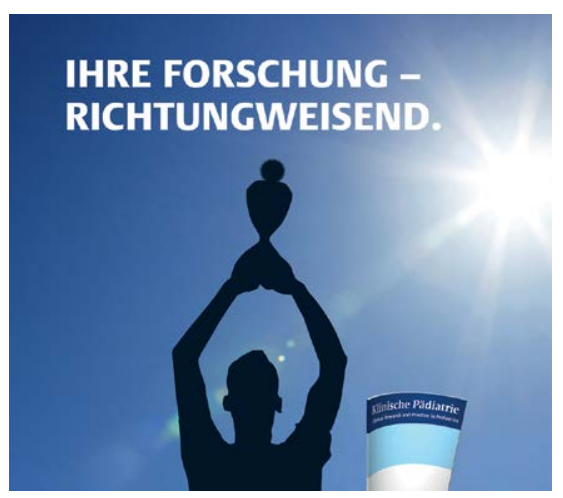

Liebe Leserinnen und Leser,

unter allen Original- und Übersichtsarbeiten, die ab dem 1. Oktober 2014 eingereicht und bis zum 30. Juni 2015 zur Publikation in der Klinischen Pädiatrie angenommen wurden, hat unsere Jury 2 herausragende Artikel ermittelt und damit erstmals den Wissenschaftspreis Klinische Pädiatrie vergeben. Bei den prämierten Artikeln handelt sich um die folgenden Beiträge:

- U. Creutzig ${ }^{1 *}$, M. Dworzak², M. Zimmermann ${ }^{1}$, J.-P. Bourquin ${ }^{3}$, B. Gruhn ${ }^{4}$, G. Fleischhack ${ }^{5}$, N. Graf ${ }^{6}$, T. Klingebiel ${ }^{7}$, B. Kremens ${ }^{5}$, T. Lehrnbecher ${ }^{7}$, C. von Neuhoff ${ }^{5}$, A. von Stackelberg ${ }^{8}$, J. Stray ${ }^{9}$, D. Reinhardt ${ }^{5}$. Randomised Introduction of 2-CDA as Intensification during Consolidation for Children with High-risk AML - Results from Study AML-BFM 2004. Klin Padiatr 2015; 227: 116-122

${ }^{1}$ Department of Pediatric Hematology and Oncology, Children's Hospital, Hannover Medical School, Hannover, Germany

${ }^{2}$ St. Anna Children's Hospital and Children's Cancer Research Institute, Department of Pediatrics, Medical University of Vienna, Vienna, Austria

${ }^{3}$ Pediatric Hematology/Oncology, Zürich, University of Zurich, Switzerland

${ }^{4}$ Department of Pediatrics, Jena University Hospital, Jena, Germany

${ }^{5}$ Paediatric Hematology and Oncology, Medical Center, University of Essen, Essen, Germany

${ }^{6}$ Paediatric Haematology and Oncology, Children's Hospital Medical Center, Homburg, Germany

${ }^{7}$ Pediatric Hematology, Oncology and Hemostaseology, University Children's Hospital of Frankfurt/ Main, Goethe-University Frankfurt/Main, Frankfurt, Germany

${ }^{8}$ Department of Pediatric Oncology/Hematology, Charité Universitätsmedizin Berlin, Berlin, Germany

${ }^{9}$ Department of Pediatric Hematology and Oncology, $2^{\text {nd }}$ Faculty of Medicine, Charles University and University Hospital Motol, Prague, Czech Republic

* einreichende und korrespondierende Autorin

- G. Stichtenoth ${ }^{1 *}$, C. Härtel ${ }^{1}$, J. Spiegler ${ }^{1}$, M. Dördelmann ${ }^{2}$, J. Möller ${ }^{3}$, C. Wieg $^{4}$, T. Orlikowsky ${ }^{5}$, A. Stein ${ }^{6}$, E. Herting ${ }^{1}$, W. Goepel ${ }^{1}$. Increased Risk for Bronchitis after Discharge in Nonvaccinated Very Low Birth Weight Infants. Klin Padiatr 2015; 227: 80-83

\footnotetext{
${ }^{1}$ Department of Pediatrics, University Hospital of Schleswig-Holstein, Lübeck, Germany

${ }^{2}$ Department of Paediatrics, Diakonissenkrankenhaus, Flensburg, Germany

${ }^{3}$ Department of Pediatrics, Saarbrücken General Hospital, Saarbrücken, Germany

${ }^{4}$ Department of Neonatology, Children's Hospital, Aschaffenburg, Germany

${ }^{5}$ Department of Neonatology, University Hospital Aachen, Aachen, Germany

${ }^{6}$ Departments of Pediatrics I, Neonatology, University Hospital, Essen, Germany

* einreichender und korrespondierender Autor
}

Mit dem Wissenschaftspreis Klinische Pädiatrie wird der besondere Beitrag dieser beiden Arbeiten zur pädiatrischen Forschung gewürdigt. Im Rahmen seiner Studie kam das Autorenteam der von Frau Professor Ursula Creutzig (Hannover) eingereichten Originalarbeit zu dem Schluss, dass sich die Prognose von Kindern und Jugendlichen mit akuter myeloischer Leukämie und hohem Risiko durch zusätzliche Gabe von moderat dosiertem 2-Chlor-2-Desoxyadenosin (2-CDA) nicht verbessert. Die prämierte Arbeit von Herrn Dr. Guido Stichtenoth (Lübeck) und Mitautoren zeigt, dass das Bronchitis-Risiko von kleinen Frühgeborenen durch zeitgerechte Impfungen reduziert werden kann.

Der Wissenschaftspreis Klinische Pädiatrie wird im Jahre 2015 für 2 herausragende Veröffentlichungen in der Pädiatrie verliehen und ist auf insgesamt 10.000 Euro dotiert. Das Preisgeld für die prämierten Artikel wird zwischen dem einreichenden Autor und seiner Institution aufgeteilt. Ziel des Wissenschaftspreises ist es, die wissenschaftliche Forschung in der Pädiatrie zu fördern und ihre Exzellenz zu würdigen. Er soll forschenden und praktisch-klinisch tätigen Pädiatern einen Anreiz bieten, ihre wissenschaftlichen Erkenntnisse in einem deutschsprachigen Umfeld weiterzugeben. Wir gratulieren den Autoren und Institutionen der prämierten Arbeiten herzlich und danken ihnen für ihren wertvollen Beitrag zu unserer Zeitschrift! 